\title{
Evidence of an Acinar Response following Treatment for Exacerbation in Adult Patients with Cystic Fibrosis
}

\author{
Eef Vanderhelst ${ }^{a, b}$ Liesbeth De Meirleir ${ }^{a, b}$ Daniel Schuermans ${ }^{a}$ \\ Anne Malfroot $^{\mathrm{b}}$ Walter Vincken ${ }^{\mathrm{a}}$ Sylvia Verbanck ${ }^{\mathrm{a}}$ \\ ${ }^{a}$ Respiratory Division and ${ }^{b}$ Cystic Fibrosis Center, University Hospital UZ Brussel, Vrije Universiteit Brussel, \\ Brussels, Belgium
}

For editorial comment see p. 459

\section{Key Words}

Adult cystic fibrosis · Infectious exacerbation - Ventilation heterogeneity $\cdot$ Lung clearance index

\begin{abstract}
Background: Treating acute infectious exacerbations in cystic fibrosis (CF) patients with intravenous antibiotic therapy leads to variability in lung function and the ventilation distribution response. Part of the variable lung clearance index $(\mathrm{LCl})$ response could be associated with the variable peripheral effects of intravenous antibiotic administration. Objectives: We explored to what extent the peripheral lung zones of CF patients could contribute to lung function improvements following treatment for infectious exacerbations. Methods: Over a 1-year period, 15 adult CF patients admitted for acute exacerbations were recruited. Lung function and multiple breath washout (MBW) tests were performed on the day of admission and at discharge. From the MBW test, we obtained acinar and conductive indices of ventilation heterogeneity and $\mathrm{LCl}$. Results: The mean age $( \pm \mathrm{SD})$ was $26 \pm 5$ years. Upon admission, the FEV 1 was $54 \pm 16 \%$ predicted and the $\mathrm{LCl}$ was $181 \pm 26 \%$ predicted. After treatment, the average $F V_{1}$ increased to $61 \pm 20 \%$ predicted $(p<0.001)$ and the $\mathrm{LCl}$ decreased to $173 \pm 28 \%$ predicted ( $p=0.042$ ). The change in $\mathrm{LCl}$ was associated with a change in acinar $(\rho=$
\end{abstract}

$+0.54 ; p=0.039)$ but not in conductive ventilation heterogeneity $(p>0.1)$. Conclusions: In CF patients in whom an improvement in $\mathrm{LCl}$ was obtained after treatment for an acute infectious exacerbation, this was paralleled by a decrease in acinar ventilation heterogeneity.

(c) 2014 S. Karger AG, Basel

\section{Introduction}

Cystic fibrosis (CF) is characterized by persistent infection and inflammation leading to progressive lung function decline. One of the hallmarks of CF is pulmonary exacerbation. The Cystic Fibrosis Foundation Clinical Practice Guidelines define a pulmonary exacerbation as the occurrence of at least 3 (out of 11 potential) new findings or changes in clinical status compared to the most recent baseline visit [1]. Potential findings or changes are: cough, sputum production, fever, weight loss, absenteeism, respiratory rate, chest examination, exercise tolerance, spirometry, oximetry, and chest radiograph. The Cystic Fibrosis Foundation's 2011 Annual Report showed that 39\% of adult CF patients were treated with intravenous antibiotics for at least 1 exacerbation per year [2]. At our center, approximately one third of the adult CF patient population in 2011 was treated with intravenous antibiotics.

\section{KARGER}

E-Mail karger@karger.com

www.karger.com/res
C 2014 S. Karger AG, Basel

0025-7931/14/0876-0492\$39.50/0 
While patients generally report a clinical improvement after a short course of intravenous antibiotic treatment, the actual effect of this treatment on the lungs has been mainly assessed in terms of spirometry (e.g. see Sanders et al. [3]). Two recent reports also made use of a global index of ventilation heterogeneity derived from a multiple-breath washout (MBW) test, such as the lung clearance index (LCI) $[4,5]$. This index was first introduced more than 60 years ago [6] and has shown promise as an early indicator of CF lung disease [7].

Robinson et al. [4] were the first to use the LCI to assess the response to intravenous antibiotics, observing considerable intersubject variability in LCI decreases (i.e. improvement) among 28 CF children (aged 8-18 years), with an average LCI decrease from 10.1 to 9.6. Horsley et al. [5] studied the impact of antibiotic treatment in 38 patients aged over 10 years from 3 different hospital centers and observed a significant LCI decrease from 14.6 to 13.8 for the group as a whole. While the final aim of this study was to identify the parameters (among 46 assays including inflammatory markers, CT imaging, and lung function parameters) that could be useful as outcome measures for the follow-up of CF patients in future clinical trials, it also confirmed the variable effect of treatment of infectious exacerbations using intravenous antibiotics on LCI.

As is the case for many other parameters derived from the MBW concentration curve, such as the moment ratio or mixing ratio $[8,9]$, the LCI cannot be attributed to proximal or peripheral lung units. Using the MBW, this can be done using a normalized phase III slope analysis, leading to indices of acinar and conductive ventilation heterogeneity [10]. We recently showed in two very different lung diseases, i.e. diffuse panbronchiolitis [11] and primary acquired pulmonary alveolar proteinosis [12], how a specific treatment of the peripheral lung zones is reflected in the response of the MBW-derived index of acinar ventilation heterogeneity. We also showed how the identification of conductive and acinar components of ventilation heterogeneity can be hampered by the severity of the lung disease, for instance in adult CF patients $[13,14]$. We showed that a combination of a large LCI value and a highly curvilinear washout curve, often observed in adult CF patients, is suggestive of ventilation heterogeneity between relatively large lung units [13]. These large-scale ventilation heterogeneities could potentially mask the more peripheral component of ventilation heterogeneity. Hence, we explored in a dedicated study [14] how the currently accepted MBW indices of conductive and acinar ventilation heterogeneity could be adapted to accurately represent ventilation heterogeneity at various lung depths, even in patients with severe lung disease.

In this prospective study, we used LCI as well as indices of conductive and acinar lung zone ventilation heterogeneity to specifically study the potential effect of antibiotic treatment administered via the intravenous route on the peripheral compartment of the adult CF lung. While intravenous antibiotic administration is the pivotal component of treatment for exacerbations, the effect of physiotherapy in the clinic cannot possibly be excluded from the treatment protocol. Based on the only two studies on the effect of physiotherapy on ventilation heterogeneity in pediatric CF patients, a consistent effect cannot be inferred $[15,16]$. Because the exact predictors of the response to antibiotic treatment in any individual patient remain elusive, we hypothesized that if there is an effect due to intravenous administration it should be most visible in the acinar component of the ventilation distribution. We also evaluated how this peripheral component of ventilation heterogeneity relates to the corresponding changes in LCI.

\section{Materials and Methods}

\section{Patients}

This prospective observational cohort trial was carried out at the Adult Cystic Fibrosis Centre of the University Hospital Brussels. This study was approved by the ethics committee (BUN143201213405) and all patients provided written informed consent. Between February 2012 and February 2013, we recruited all consecutive adult CF patients who were admitted for an acute pulmonary exacerbation by the hospital CF team for a 10-day course of intravenous antibiotic therapy (allowing also for repeat visits); the diagnosis of an exacerbation was based on the criteria of the 1997 Clinical Practice Guidelines of the Cystic Fibrosis Foundation [1]. The choice of antibiotics was based on the individual antibiotic susceptibility results of the most recent respiratory tract sample. A combination of at least two different antibiotics was given.

\section{Measurements}

After having completed their daily standard airway clearance therapy - performed by the CF physiotherapist - patients were invited to the pulmonary function lab, where spirometry, plethysmography, and MBW tests were performed after bronchodilation with $400 \mu \mathrm{g}$ salbutamol (via a Volumatic ${ }^{\circledR}$ spacer). This was done at baseline (less than $24 \mathrm{~h}$ following the initiation of intravenous antibiotics) and 8 days later. On the same days, blood samples were obtained from which inflammatory parameters, i.e. white blood cell counts and C-reactive protein levels, were determined.

All lung function measurements were performed by the same lung function technician and according to the standard ERS guidelines for lung function measurements [17]. MBW testing and anal- 
yses were done according to instructions extensively described in a recent standardization paper [18]. The MBW test is performed via 1-liter tidal breathing of pure oxygen while the nitrogen concentration is being measured as a function of the cumulative expired volume. The MBW equipment used had remained unchanged since its original use [10]. The reference values for the standard parameters of global ventilation heterogeneity (LCI) and acinar and conductive ventilation heterogeneity (Sacin and Scond) obtained with a semiautomated phase III slope determination can be found in the study of Verbanck et al. [19]. Besides the LCI, we also computed another measure of global ventilation heterogeneity (Curv) from the mean expired concentration washout curve. Curv equals 1 minus the ratio of the regression slopes between lung turnover LCI/2 and LCI, and between 0 and LCI/2 [13]. This measure of curvature is slightly different from that originally used by us and others (e.g. see Verbanck et al. [10] and Prisk et al. [20]) in that it is 1 minus the ratio instead of the ratio itself, so as to have an increase in the Curv value with increasing the curvature. Computed in this way, Curv $=0$ reflects a homogeneous ventilation and Curv $=1$ corresponds to the presence of an infinitesimally slowly emptying lung unit; in normal subjects, Curv is approximately 0.2 $[13,21]$.

We also performed a normalized phase III slope analysis of the MBW test. In the standard method, conductive ventilation heterogeneity (Scond) is computed as the regression slope of the normalized phase III slopes between lung turnovers 1.5 and 6 [10]. In a modified analysis tailored to include severe CF lung disease [14], conductive ventilation heterogeneity (Scond*) is computed as the regression slope of the normalized phase III slopes, between lung turnovers 0 and 3, and excluding the first breath. Corresponding Sacin (or Sacin*) values are obtained by subtracting from the normalized slope of the first breath, Scond (or Scond*), multiplied by the lung turnover of the first breath. Results from both analyses are reported here.

\section{Statistical Analysis}

All statistical analyses were performed using MedCalc (version 10.4; Mariakerke, Belgium). All variables were tested for normality using the $\chi^{2}$ test. Depending on the normality, each parameter was tested for antibiotic treatment changes with respect to baseline by means of paired t tests or Wilcoxon's paired tests. Associations between treatment-induced changes in the various parameters were tested using Spearman's correlations in order to not make any assumptions about the linearity of these relationships. $\mathrm{p}<0.05$ was considered statistically significant.

\section{Results}

Over the 1-year recruitment period, 23 single adult CF patients were admitted to the hospital for an exacerbation, constituting $28 \%$ of the adult CF population at our center. Two patients were excluded because of pregnancy, 1 patient was not able to perform on lung function tests due to mental retardation, and 1 patient was excluded because she was on the lung transplant list. Data from another 4 patients could not be used because of premature discharge $(n=1)$ or an unforeseen continuation of intravenous therapy at home $(n=3)$. The breakdown of the cohort is shown in the supplementary material (for all online suppl. material, see www.karger.com/ doi/10.1159/000360772). Fifteen patients, 6 of whom were admitted for an intravenous antibiotics course on more than one occasion (2-4 times) during the 1-year recruitment period, completed the study. The mean age $( \pm \mathrm{SD})$ of the patients was $26 \pm 5$ years, and the mean BMI was $19.9 \pm 2.6$. All 15 patients had pancreatic insufficiency, 5 out of 15 patients suffered from CF-related diabetes, 67\% (10 patients) were chronically colonized with Pseudomonas aeruginosa, and all patients had bronchiectases. Five of the 15 patients were homozygotous for the genotype F508del.

Averaged over all admissions for the 15 patients, the baseline and postantibiotic (post $\mathrm{AB}$ ) lung function measurements were performed, respectively, $0.4 \pm 0.7$ and $8.1 \pm 1.5$ days after the actual onset of intravenous antibiotics. For the 6 patients with multiple visits, we first selected the data of each patient's first visit. As a result of this selection, data from 15 single visits of 15 single study patients were considered for all further analyses. Table 1 shows the clinical parameters, standard lung function values, and MBW indices obtained at baseline and post$\mathrm{AB}$. The standard lung function showed modest but statistically significant increases in $\mathrm{FEV}_{1}, \mathrm{FVC}, \mathrm{FEV}_{1} / \mathrm{FVC}$, and $\mathrm{FEF}_{75}$ postAB for the group as a whole. There was also a small but significant increase in TLC (3.3\% predicted) but no change in RV/TLC ( $\mathrm{p}>0.1)$.

Table 1 also shows that the CF patients under study had severe ventilation heterogeneity at baseline (at the onset of antibiotic treatment), specifically with Curv values in excess of 0.47 , a cutoff which signals the use of the modified indices Scond* and Sacin* instead of Scond and Sacin [14]. Following antibiotic treatment, the indices of ventilation heterogeneity specifically sensitive to conductive ventilation heterogeneity (Curv and Scond or Scond*) did not show improvement for the group as a whole. By contrast, Sacin (and Sacin*) significantly decreased (by $18.5 \%$ for both). The global parameter of ventilation heterogeneity LCI also showed a significant decrease (4.5\%). The average ventilated FRC (FRC $\left.{ }_{\mathrm{MBW}}\right)$ decreased by 138 $\mathrm{ml}$, constituting $5 \%$ of the baseline FRC. Finally, the difference between thoracic and ventilated FRC $\left(\mathrm{FRC}_{\text {pleth }}-\right.$ $\left.\mathrm{FRC}_{\mathrm{MBW}}\right)$ did not change postAB ( $\left.\mathrm{p}>0.1\right)$.

In order to explore whether the observed LCI changes were the result of changes in the acinar and/or conductive components of ventilation heterogeneity [20], a correlation analysis was performed including LCI, Sacin*, and 
Table 1. Laboratory and lung function parameters before and after antibiotic therapy

\begin{tabular}{|c|c|c|c|c|c|}
\hline & \multicolumn{2}{|c|}{ Baseline } & \multicolumn{2}{|c|}{ PostAB } & \multirow[t]{2}{*}{$\mathrm{p}$ value } \\
\hline & mean $^{\mathrm{a}}$ & $\mathrm{SD}^{\mathrm{a}}$ & mean $^{\mathrm{a}}$ & $\mathrm{SD}^{\mathrm{a}}$ & \\
\hline $\mathrm{CRP}, \mathrm{mg} / \mathrm{l}$ & 16.1 & $(8-44)$ & 7 & $(7-15)$ & $<0.001$ \\
\hline White blood cells, $\mathrm{n} \times 10^{3} / \mathrm{mm}^{3}$ & 10.2 & 3.9 & 8.1 & 2.8 & 0.025 \\
\hline \multicolumn{6}{|l|}{ Lung function } \\
\hline $\mathrm{FEV}_{1}, \%$ predicted & 54 & 16 & 61 & 20 & $<0.001$ \\
\hline FVC, \% predicted & 83 & 14 & 87 & 14 & 0.0001 \\
\hline $\mathrm{FEV}_{1} / \mathrm{FVC}(\%)$ & 65 & $(41-67)$ & 67 & $(44-70)$ & 0.011 \\
\hline $\mathrm{FEF}_{75}, \%$ predicted & 11 & $(7-15)$ & 16 & $(8-24)$ & 0.017 \\
\hline RV/TLC (\%) & 39 & 12 & 37 & 10 & $>0.1$ \\
\hline TLC, $\%$ predicted & 97 & 16 & 100 & 14 & 0.02 \\
\hline \multicolumn{6}{|l|}{$M B W$} \\
\hline $\mathrm{FRC}_{\mathrm{MBW}}, \mathrm{ml}$ & 2,798 & 793 & 2,661 & 744 & 0.017 \\
\hline $\mathrm{FRC}_{\text {pleth }}-\mathrm{FRC}_{\mathrm{MBW}}, \mathrm{ml}$ & 947 & $537-1,461$ & 819 & $767-1,592$ & $>0.1$ \\
\hline LCI & 10.6 & 1.5 & 10.1 & 1.6 & 0.045 \\
\hline LCI (\% predicted) & 181 & 26 & 173 & 28 & 0.042 \\
\hline Curv & 0.58 & 0.08 & 0.59 & 0.10 & $>0.1$ \\
\hline $\operatorname{Sacin}\left(1^{-1}\right)$ & 0.44 & 0.22 & 0.36 & 0.19 & 0.01 \\
\hline Scond $\left(1^{-1}\right)$ & 0.104 & 0.022 & 0.107 & 0.026 & $>0.1$ \\
\hline Sacin $*\left(1^{-1}\right)$ & 0.41 & 0.22 & 0.33 & 0.18 & 0.013 \\
\hline Scond* $\left(\mathrm{l}^{-1}\right)(\mathrm{ml})$ & 0.177 & 0.027 & 0.169 & 0.034 & $>0.1$ \\
\hline
\end{tabular}

$\mathrm{RV}=$ Residual volume; Scond and Sacin = standard indices of conductive and acinar ventilation heterogeneity derived from normalized phase III slopes; Scond* and Sacin* = adaptions of Scond and Sacin for adult CF patients (see text for details).

${ }^{a}$ For parameters failing the normality test, medians and 95\% CI are provided instead of means and SD, and Wilcoxon's tests instead of paired t tests were used to test for significant changes between baseline and post $\mathrm{AB}$.

Scond*. LCI was correlated with Sacin* $(\rho=0.54 ; \mathrm{p}=$ $0.039)$ but not with Scond* $(\rho=0.14 ; p>0.1)$. A graphical representation of the relationship between LCI and Sacin* at baseline, and their respective changes post $A B$, is shown by the connected symbols in figure 1 . In order to assess whether the observed LCI and Sacin* changes postAB could be attributed to the decrease in ventilated FRC, the correlations with $\mathrm{FRC}_{\mathrm{MBW}}$ were also examined. In fact, $\mathrm{FRC}_{\mathrm{MBW}}$ changes did not correlate with any of the indices of ventilation heterogeneity. The correlations between all parameters measured (table 1) can be found in the online supplementary material.

Figure 2 displays the Sacin* and LCI changes found in 6 CF patients each time they were readmitted for intravenous antibiotics. Though they constitute a small group, these data illustrate the similarity across visits of the baseline and post $\mathrm{AB}$ changes in LCI and Sacin*, at least within this 1-year time frame.

In the online supplementary material, the MBW concentration and normalized phase III slope curves from which the MBW parameters Scond and Sacin (or Scond* and Sacin*) are derived are shown for 2 representative CF patients. Both patients display the hallmark of an acinar lung zone effect post $\mathrm{AB}$, and one patient illustrates an additional conductive effect. From these curves it can also be appreciated how Scond underestimates the actual degree of conductive ventilation heterogeneity in the CF patients under study and why Scond* (and Sacin*) is to be preferred over Scond (and Sacin). Also, the Sacin, Sacin*, Scond, Scond*, and LCI values of the CF patients under study here are plotted against values previously obtained in a wider population of stable $\mathrm{CF}$ patients representative of our center [14].

\section{Discussion}

The present study shows that acinar rather than conductive ventilation heterogeneity constitutes the main response to treatment for acute infectious exacerbations - 


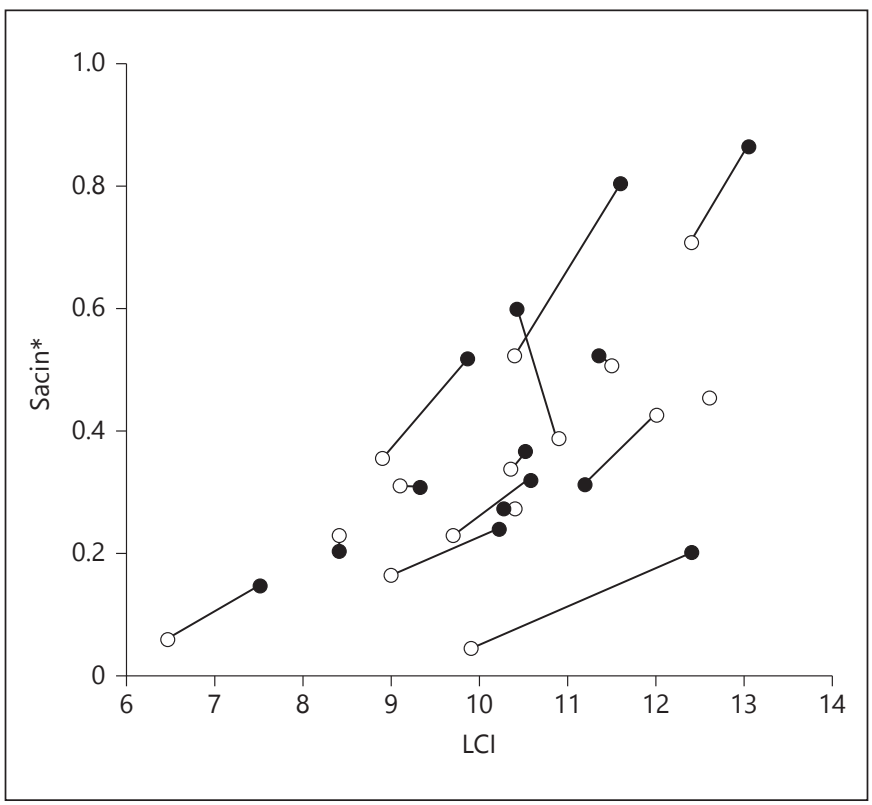

Fig. 1. Baseline and post $A B$ values of Sacin* $\left(1^{-1}\right)$ vs. corresponding LCI values obtained in 15 CF patients; connected circles indicate data pairs of baseline (closed circles) vs. postAB (open circles) values.

including intravenous antibiotic administration - in adult CF. Because the intravenous route of antibiotic administration was the only aspect of the in-clinic treatment for exacerbation which was not a part of the maintenance treatment at home (prior to admission), it is tempting to speculate on its specific effect based on the mechanisms generating ventilation heterogeneity in adult CF lung disease. We have previously shown that relatively large convection-dependent units are a major determinant of ventilation heterogeneity in adult CF patients with high LCI and Curv values [13]. Given the high LCI and Curv values observed in the CF patients under study (table 1), it is unlikely that one course of intravenous antibiotic treatment would have resolved any such large scale changes. One could speculate that clearance of the mucus partially obstructing the relatively large airways which feed the convective flow into subtended lung zones could modify some of the convection-dependent ventilation heterogeneities or open up ventilated air spaces. However, the absence of significant changes in Curv and Scond* after antibiotic treatment (table 1) does not support this. Also, the slight FRC decrease observed here (table 1) suggests that the total volume of ventilated lung opening up in our patients must have been limited and in any case insufficient to counteract what is probably a small reduction in hyperinflation after treatment. Finally, the trapped gas quanti- fied by the difference between thoracic and ventilated gases at FRC, which was considerable in these patients (in the order of 1 liter), was unaffected by treatment.

Gozal et al. [22] were the first to point out that tests of ventilation heterogeneity (and, in their case, the singlebreath washout phase III slope) are a very sensitive noninvasive tool to gauge antibiotic treatment. They also pointed out that indices of ventilation heterogeneity can lead to counterintuitive increases, potentially reflecting ventilation heterogeneity over a different part of the lungs than at baseline. Along these lines, one could assume that mucus plugs both in large and small airways would introduce some variability in the baseline condition of ventilation heterogeneity or in the patient's response to antibiotics. The baseline and postAB values of LCI (and Sacin*) obtained in patients who returned for exacerbation treatment suggest that, in the face of intersubject variability in the response to intravenous antibiotics, the intrasubject variability is considerably less (fig. 2). When a patient shows a response, this appears to be the case on several occasions at least within a 1-year time span; the same holds true when a patient does not show a response. The similar intrasubject baseline Sacin* and LCI values do not appear to reflect the variable effect one might expect of mucus plugs, unless these are harbored in similar locations upon every exacerbation. Also, the consistency of the response (or nonresponse) in terms of Sacin* and LCI across several exacerbations in the same patient suggests a very similar lung functional unit being tackled for improvement.

The degree of baseline LCI abnormality in CF patients with an exacerbation and the LCI decrease postAB observed here were similar to those obtained by Horsley et al. [5] and Robinson et al. [4]. Together, our studies indicate that, when research conditions can ensure good quality LCI data, the LCI could be used as an outcome parameter, bearing in mind that some patients will show a lung functional response while others simply will not. We have shown here that when an LCI decrease is observed following treatment for an acute exacerbation including intravenous antibiotics, it is predominantly obtained by an acinar effect likely due to the administration route which reaches the most peripheral lung zones.

The treatment of exacerbations usually involves intravenous or oral antibiotics and intensification of physiotherapy with sputum clearance. Currently, there is no evidence to support the use of inhaled antibiotics in an acute exacerbation [23]. This is based on the rationale that, during an acute exacerbation, an increased production of mucus plugs could obstruct the conductive airways, pre- 


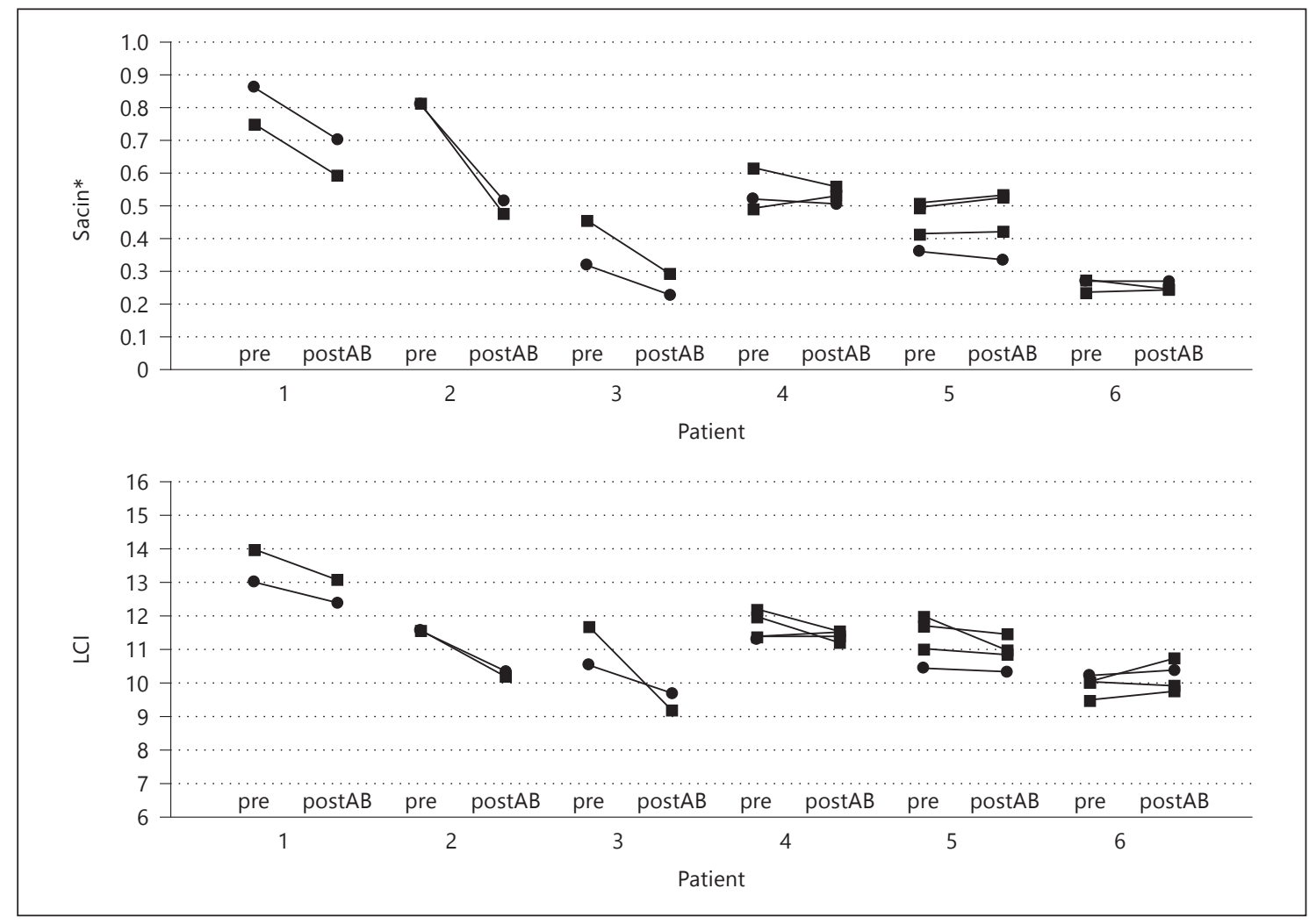

Fig. 2. Sacin* $\left(1^{-1}\right)$ and LCI values obtained in 6 CF patients who were readmitted for a pulmonary exacerbation on several occasions. Patients 1-3 showed a response and did so on every occasion; patients 4-6 did not show a response and did not do so on any occasion. The first visit for each patient is indicated by closed circles. pre $=$ Baseline.

venting the inhaled antibiotics from reaching the deeper areas of the respiratory tract. In our study group, 12 out of 15 patients received inhaled antibiotics as maintenance treatment, which was continued during the in-hospital intravenous therapy. In these patients, the effect on lung function after intravenous antibiotics can indeed be considered an add-on effect. In-hospital intravenous treatment has been shown to have a greater effect on $\mathrm{FEV}_{1}$ than treatment at home [24], which could also be partly attributed to the concomitant regular regime of physiotherapy and nutrition provided in-hospital.

The main limitation of this study was the relatively small number of eligible adult CF patients that could be recruited over a 1-year period, because we aimed to restrict our study to a single center where tightly controlled in-hospital conditions could be guaranteed. Another limitation intrinsic to this type of study by us and others is the fact that the effect of physiotherapy and the increased adherence to other maintenance therapies (e.g. DNAse) in the clinic cannot be excluded. To the best of our knowl- edge, no clear-cut effects of physiotherapy on ventilation distribution have been demonstrated. Fuchs et al. [15] did not observe any consistent effects of physiotherapy on the LCI or on the ventilated FRC. While they did not aim to study the efficacy of physiotherapy per se, Abbas et al. [16] did observe some changes in single-breath-washoutderived indices following inhalation therapy and following subsequent physiotherapy; the potential associated FRC changes that could have affected these indices were not documented. Even if our observed decrease in ventilated $\mathrm{FRC}$ (by 5\%; $\mathrm{FRC}_{\mathrm{MBW}}$ in table 1) were attributable to physiotherapy, the absence of a correlation of this FRC change with any other index under study supports the fact that the improvement in the acinar compartment reflects an independent effect. Finally, we need to consider that both of the above mentioned physiotherapy studies dealt with pediatric CF patients, while the adult CF patients studied here generally have extensive experience with physiotherapy as part of their daily routine. Hence, the specific add-on effect of physiotherapy in the clinic 
should probably not be exaggerated. Despite the recommendation by Fuchs et al. [15], i.e. that the timing of the MBW with respect to physiotherapy can thus be ignored, we attempted to obtain the MBW data in a standardized way, at the end of the same test sequence. By performing baseline and post $A B$ lung function measurements after physiotherapy and bronchodilation, the variability in the study outcome measures was minimized.

In conclusion, we identified the peripheral lung zones where short-term treatment with intravenous antibiotics for an acute infectious exacerbation can elicit a re- sponse. The greatest beneficial effect of the treatment was manifested as a decrease in ventilation heterogeneity in the acinar lung zone, with an associated decrease in the overall measure of ventilation heterogeneity, i.e the LCI. In responsive patients, in whom the LCI and acinar ventilation heterogeneity could be improved, this was observed to repeat within a 1-year observation window. To our knowledge, this is the first study to examine and report such peripheral changes in a lung disease that is burdened by ventilation heterogeneity at various lung depths.

\section{References}

1 Clinical Practice Guidelines for Cystic Fibrosis. Bethesda, Cystic Fibrosis Foundation, 1997.

2 Cystic Fibrosis Foundation: UK Patient Registry Annual Report 2011. Bethesda, Cystic Fibrosis Foundation, 2011.

-3 Sanders DB, Bittner RC, Rosenfeld M, Hoffman LR, Redding GJ, Goss CH: Failure to recover to baseline pulmonary function after cystic fibrosis pulmonary exacerbation. Am J Respir Crit Care Med 2010;182:627-632.

4 Robinson PD, Cooper P, Van Asperen P, Fitzgerald D, Selvadurai H: Using index of ventilation to assess response to treatment for acute pulmonary exacerbation in children with cystic fibrosis. Pediatr Pulmonol 2009; 44:733-742.

5 Horsley AR, Davies JC, Gray RD, Macleod KA, Donovan J, Aziz ZA, Bell NJ, Rainer M, Mt-Isa S, Voase N, Dewar MH, Saunders C, Gibson JS, Parra-Leiton J, Larsen MD, Jeswiet S, Soussi S, Bakar Y, Meister MG, Tyler P, Doherty A, Hansell DM, Ashby D, Hyde SC, Gill DR, Greening AP, Porteous DJ, Innes JA, Boyd AC, Griesenbach U, Cunningham S, Alton EW: Changes in physiological, functional and structural markers of cystic fibrosis lung disease with treatment of a pulmonary exacerbation. Thorax 2013;68:532-539.

6 Becklake MR: A new index of the intrapulmonary mixture of inspired air. Thorax 1952;7: $111-116$.

7 Kraemer R, Blum A, Schibler A, Ammann RA, Gallati S: Ventilation inhomogeneities in relation to standard lung function in patients with cystic fibrosis. Am J Respir Crit Care Med 2005;171:371-378.

8 Engel LA: Intraregional gas mixing and distribution; in Engel LA, Paiva M (eds): Gas Mixing and Distribution in the Lung. New York, Marcel Dekker, 1985, pp 287-358.

-9 Larsson A, Jonmarker C, Werner O: Ventilation inhomogeneity during controlled ventilation: which index should be used? J Appl Physiol 1988;65:2030-2039.
0 Verbanck S, Schuermans D, Van Muylem A, Paiva M, Noppen M, Vincken W: Ventilation distribution during histamine provocation. J Appl Physiol 1997;83:1907-1916.

-11 Hanon S, Verbanck S, Schuermans D, Vanden Berghe B, Vanderhelst E, Vincken W: Evidence of improved small airways function after azithromycin treatment in diffuse panbronchiolitis. Respiration 2012;84:75-79.

12 Vanderhelst E, Hanon S, Verbanck S, Schuermans D, Wissing K, Bonella F, Vincken W: Whole-lung lavage: a successful treatment for restoring acinar ventilation distribution in primary acquired pulmonary alveolar proteinosis. Respiration 2012;84:70-74.

13 Verbanck S, Paiva M, Paeps E, Schuermans D, Malfroot A, Vincken W, Vanderhelst E: Lung clearance index in adult CF patients: the role of convection-dependent lung units. Eur Respir J 2013;42:380-388.

14 Verbanck S, Paiva M, Schuermans D, Malfroot A, Vincken W, Vanderhelst E: Acinar and conductive ventilation heterogeneity in severe CF lung disease: back to the model. Respir Physiol Neurobiol 2013;188:124-132.

15 Fuchs SI, Toussaint S, Edlhaimb B, Ballmann M, Gappa M: Short-term effect of physiotherapy on variability of the lung clearance index in children with cystic fibrosis. Pediatr Pulmonol 2010;45:301-306.

-16 Abbas C, Singer F, Yammine S, Casualta C, Latzin P: Treatment response of airway clearance assessed by single breath washout in children with cystic fibrosis. J Cyst Fibros 2013;12:567-574.
Pellegrino R, Viegi G, Brusasco V, Crapo RO, Burgos F, Casaburi R, Coates A, van der Grinten CP, Gustafsson P, Hankinson J, Jensen R, Johnson DC, MacIntyre N, McKay R, Miller MR, Navajas D, Pedersen OF, Wanger $\mathrm{J}$ : Interpretative strategies for lung function tests. Eur Respir J 2005;26:948-968.

18 Robinson PD, Latzin P, Verbanck S, Hall GL, Horsley A, Gappa M, Thamrin C, Arets HG, Aurora P, Fuchs SI, King GG, Lum S, Macleod K, Paiva M, Pillow JJ, Ranganathan S, Ratjen F, Singer F, Sonnappa S, Stocks J, Subbarao P, Thompson BR, Gustafsson PM: Consensus statement for inert gas washout measurement using multiple- and single-breath tests. Eur Respir J 2013;41:507-522.

19 Verbanck S, Thompson BR, Schuermans D, Kalsi H, Biddiscombe M, Stuart-Andrews C, Hanon S, Van Muylem A, Paiva M, Vincken $\mathrm{W}$, Usmani O: Ventilation heterogeneity in the acinar and conductive zones of the normal ageing lung. Thorax 2012;67:789-795.

20 Prisk GK, Elliott AR, Guy HJ, Verbanck S, Paiva M, West JB: Multiple breath wash in of helium and sulfur hexafluoride in sustained microgravity. J App Physiol 1998;84:244-252.

21 Verbanck S, Paiva M, Schuermans D, Hanon S, Vincken W, Van Muylem A: Relationships between the lung clearance index and conductive and acinar ventilation heterogeneity. J Appl Physiol 2012;112:782-790.

22 Gozal D, Bailey S, Keens T: Evolution of pulmonary function during an acute exacerbation in hospitalized patients with cystic fibrosis. Pediatr Pulmonol 1993;16:347-353.

23 Döring G, Flume P, Heijerman H, Elborn JS; Consensus Study Group: Treatment of lung infection in patients with cystic fibrosis: current and future strategies. J Cyst Fibros 2012; 11:461-479.

24 Thornton J, Elliott R, Tully MP, Dodd M, Webb AK: Long term clinical outcome of home and hospital intravenous antibiotic treatment in adults with cystic fibrosis. Tho$\operatorname{rax} 2004 ; 59: 242-246$. 